\title{
Cosmic rays as an indicator of the geoeffectiveness of magnetic clouds
}

\author{
Anastasia Petukhova ${ }^{1,1}$, Ivan Petukhov ${ }^{1}$, Stanislav Petukhov ${ }^{1}$, Petr Gololobov ${ }^{1}$ \\ ${ }^{1}$ Yu.G. Shafer Institute of Cosmophysical Research and Aeronomy, SB RAS, 31 Lenin Ave., \\ Yakutsk, Russia
}

\begin{abstract}
Geomagnetic storms are initiated by organized magnetic structures of the solar wind. The intensity of magnetic storms is determined by the product of the southward component of the magnetic field and the time interval, during which the structure is located near Earth: the larger the product, the higher the storm intensity. To determine the local properties of the structures, direct spacecraft measurements of the plasma and magnetic field characteristics are used. Global properties of the structures are also of great interest. Such information can be obtained using measurements of cosmic rays by the worldwide network of neutron monitors. Magnetic clouds are examples of these structures. About $30 \%$ of magnetic storms are caused by magnetic clouds. In our theory of the formation of Forbush decrease in a magnetic cloud, it has been found that the components of the vector anisotropy in time are determined by the magnetic cloud type. Thus, using the cosmic ray method, it is possible to determine a connection between the magnetic cloud type and the intensity of the magnetic storm. Similar connections can be made for other magnetic structures.
\end{abstract}

\section{Introduction}

Interplanetary coronal mass ejections (ICMEs) strongly influence the properties of the solar wind plasma, geomagnetic activity, and the space distribution of cosmic rays (CRs). ICMEs with a super-Alfven velocity produce the shock front. There is a shock-sheath region between the shock front and the leading edge of ICMEs, where the energy densities of the regular and turbulent magnetic fields significantly increase [1]. Magnetic clouds (MCs) are a part of ICMEs. An MC is a large-scale magnetic loop rooted at the Sun [2]. The unique property of an MC is the helical structure of its magnetic field (MFR). About one-third of ICMEs at 1 au demonstrate clear features of MCs [3, 4]. Apparently, this is due to the ratio between the areas of the MC cross-section size and ICMEs. It is believed that the majority of, and maybe even all, ICMEs, have MCs [5]. According to the definitions introduced by Bothmer, \& Schwenn [4], there are the following MC types: south-west-north (SWN), south-east-north (SEN), north-west-south (NWS), and north-east-south (NES).

Determining the MC type and its orientation in interplanetary space is crucial for studying the MC effect on geomagnetic activity $[1,6]$. It has been empirically shown that

\footnotetext{
${ }^{1}$ Corresponding author: petukhova@ikfia.ysn.ru
} 
intense storms with peak Dst $\leq-100 \mathrm{nT}$ are primarily caused by large $\mathrm{Bz} \leq-10 \mathrm{nT}$ fields with duration greater than 3 hours [7]. To determine the MC properties, the results of direct spacecraft measurements are mainly used $[8,9]$. Due to their high mobility, CRs contain information about global properties of MCs and thus may provide information that cannot be locally measured [10,11]. Forbush decreases (FDs) are observed decreases in the counting rate of $\mathrm{CR}$ detectors (usually neutron monitors and muon telescopes) accompanying the passage of ICMEs through the observation point (Earth). FDs are useful indicators of ICMEs. A statistical study by Richarson and Cane [12] showed that $80 \%$ of over 300 ICMEs were accompanied by FDs. About $30 \%$ of magnetic storms are caused by magnetic clouds [13]. FDs can be particularly useful for identifying ICMEs when limited magnetic field and plasma measurements are available.

It was found that the time behaviour of the vector anisotropy components depends on the magnetic field polarization in the MC [14]. It confirms the possibility of determining the MC properties by ground-based CR measurements.

It is usually believed that FD is formed by the shock sheath region, which prevents CR penetration into ICME. Petukhova et al. [15] found that FD in an MC is formed due to cosmic energy losses. This article presents the calculation results of the FD characteristics in an MC based on the new theory, which can be used to determine a MC type using ground-based CR measurements.

\section{MC model}

The theory of the FD formation in an MC is published in [15]. In the theory, the MC model has the form of a moving loop with a helical field. Figure 1 shows the form of the loop by three magnetic field lines in an MC at $1 \mathrm{au}$.

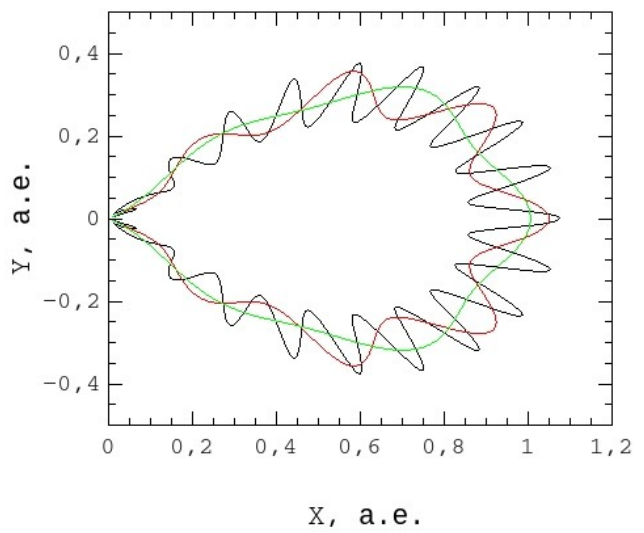

Fig. 1. Three magnetic field lines located at different distances from the MC surface for one time moment.

The CR distribution function without scattering is determined by solving the Boltzmann equation. It follows from the equation that the density of the distribution function is preserved along the equation characteristics, which are the particle trajectories. From the calculations it was found that the FD formation mechanism is the CR energy loss in an MC.

We calculated the FD amplitude, the components of the vector and tensor anisotropies along the trajectory of the MC passing Earth. It is shown that the FD characteristics depend on the MC parameters: the magnetic field strength, helical field structure, velocity and velocity gradient, geometric dimensions. It was found that the FD characteristics mainly depend on the magnetic field strength and the global helical structure of the magnetic field. 
In the new theory the FD characteristics are determined by the values of the physical parameters of the MC. There are no free parameters in the theory.

Figures $2 \mathrm{a}, 2 \mathrm{~b}$ show the calculation results of the FD amplitude and anisotropy components in time.
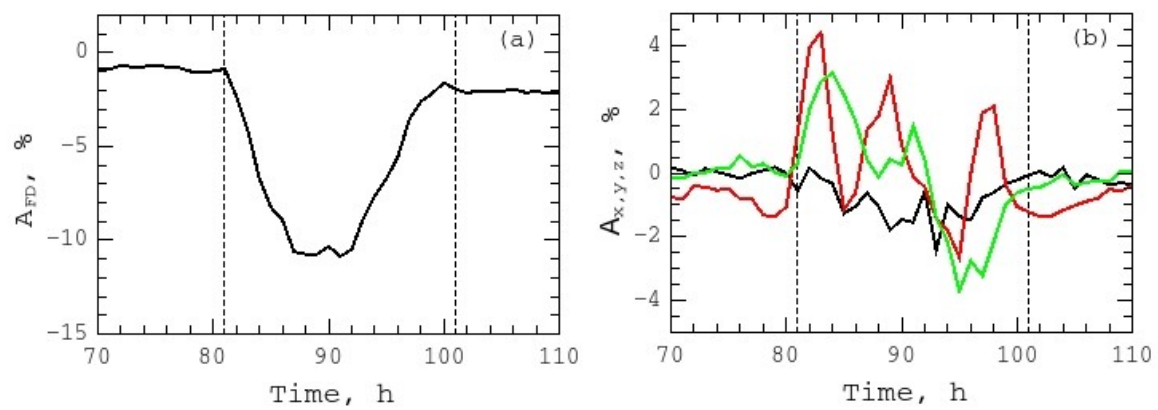

Fig. 2. (a) FD amplitude, (b) anisotropy components in time. The black, red, and green curves correspond to the Ax,y,z anisotropy components. Vertical lines denote the $\mathrm{MC}$ edges. The MC parameters at $1 \mathrm{au}$ : the velocities of the MC leading and trailing edges are $500 \mathrm{~km} \mathrm{~s}^{-1}$ and $400 \mathrm{~km} \mathrm{~s}^{-1}$, the magnetic field strength at the MC axis is $20 \mathrm{nT}$.

\section{Calculation results and conclusion}

Figures $3 \mathrm{~b}, 3 \mathrm{~d}, 3 \mathrm{f}, 3 \mathrm{~h}$ show the components of unidirectional anisotropy in time. MC type determines the behavior of the anisotropy components. For example, the magnetic field components presented in Figures $3 \mathrm{a}$ and $3 \mathrm{c}$ are related by mirror symmetry. The anisotropy components shown in Figures $3 \mathrm{~b}$ and $3 \mathrm{~d}$ are also connected by mirror symmetry. The anisotropy components shown in Figures $3 \mathrm{f}$ and $3 \mathrm{~h}$ and have a similar symmetry. The calculation results show that the components of the anisotropy Ay, Az are mirror symmetric to the components of the magnetic field $\mathrm{Bz}, \mathrm{By}$. 

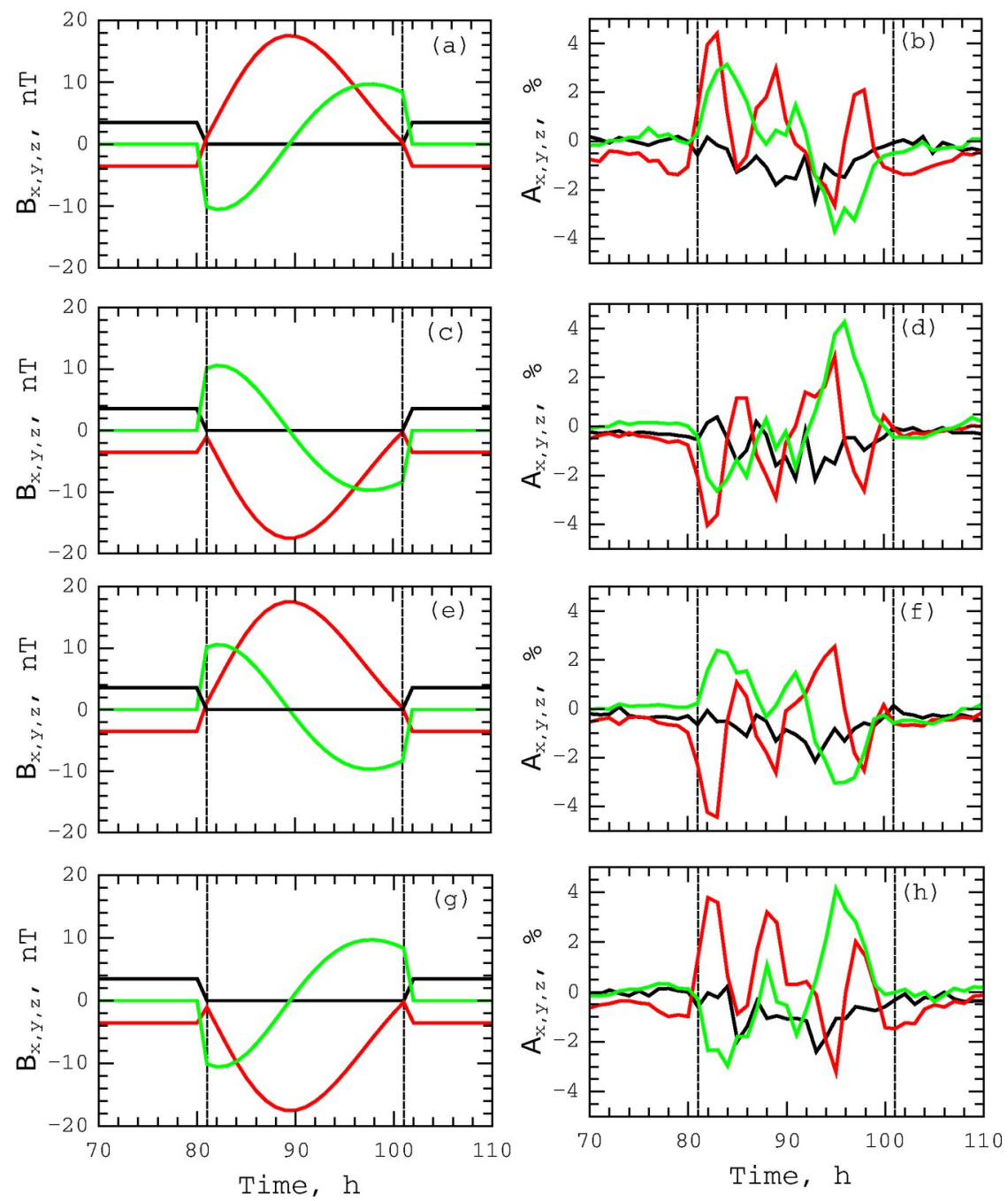

Fig. 3. (a, c, e, and g) Magnetic field components for SWN, NES, NWS, SEN types in time. The black, red, and green curves correspond to the Bx,y,z magnetic field components, respectively. (b, d, f, and h) Unidirectional anisotropy components in time. The anisotropy components in (b), (d), (f), and (h) correspond to the MC types in (a), (c), (e), and (g). The black, red, and green curves correspond to the Ax,y,z anisotropy components.

The presented calculation results show that the FD amplitude weakly depends on MC type, however, the anisotropy strongly does. The difference can be used to determine a MC type using ground-based measurements of CRs. Since the intensity of geomagnetic storms depends on MC type, then the MC geoefficiency can be determined from $\mathrm{CR}$ measurements.

Figures $4 \mathrm{a}, 4 \mathrm{~b}$ present the measurements of the magnetic field components in an MC, the FD amplitude and the anisotropy components in time for the event on 2000 July 16. The measurements of the magnetic field show that the MC type is SWN and Earth does not 
cross the axis of the loop. It can be seen from the comparison of the calculated and observed anisotropies that the behavior of Ay-components is similar, but Ax,z are not. The difference may be explained by the fact that in the calculation the impact parameter during Earth crossing the MC is zero but the impact parameter for the event is not (compare Figures $3 \mathrm{a}$ and $4 \mathrm{a}$ ). To find a relation between MC type and anisotropy components for real events, it is necessary to carry out calculations with different impact parameters.
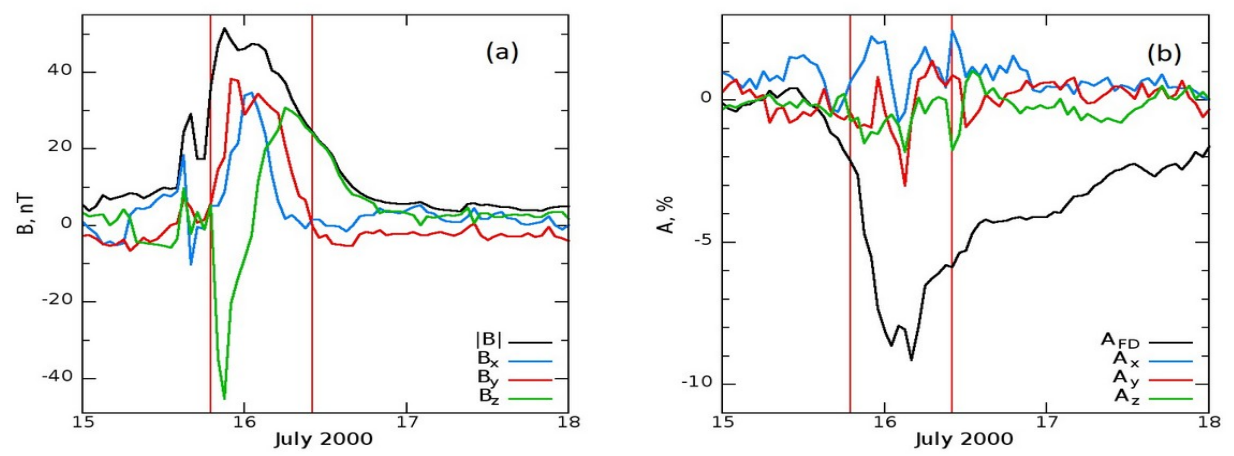

Fig. 4. (a) Module and components of magnetic field strenght, (b) FD amplitude and the anisotropy components in time for the event on 2000 July 16 . Vertical lines denote the MC edges.

This work is partially supported by the Ministry of Education and Science of the Russian Federation and the Siberian Branch of the Russian Academy of Sciences (Project II.16.2.2.). The research was partially supported by Russian Foundation for Basic Research (Project N 18-32-00064).

\section{References}

1. E. Kilpua, H. E. J.Koskinen, T. I. Pulkkinen, LRSP, 14, 5 (2017)

2. T. H. Zurbuchen, I. G. Richardson, SSRv, 123, 31 (2006)

3. J. T.Gosling, GMS, 58, 343 (1990)

4. V. Bothmer, R.Schwenn, AdSpR, 17, 319 (1996)

5. A. Vourlidas, B. J. Lynch, R. AHoward, et al., SoPh, 284, 179 (2013)

6. R. Schwenn, A. dal Lago, E. Huttunen, et al., AnGeo, 23, 1033 (2005)

7. W. D.Gonzalez, B. T. Tsurutani, Planet. Space Sci., 35, 1101 (1987)

8. L. F. Burlaga, J. Geophys. Res., 93, 7217 (1988)

9. R. P.Lepping, L. F. Burlaga, B. T. Tsurutani, et al., JGR, 96, 9425 (1991)

10. J. A. Lockwood, SSRv, 12, 658 (1971)

11. K. Munakata, T. Kuwabara, J. W. Bieber, et al., AdSpR, 36, 2357 (2005)

12. I. G. Richardson, H. V.Cane, SoPh, 270, 609 (2011)

13. J.-Ch. Zhang, M.W. Liemohn, J.U. Kozyra, et al., J. Geophys. Res. 109, A09101 (2004) 
14. A.S. Petukhova, I.S. Petukhov, and S.I. Petukhov, J. Geophys. Res., 124, 19-31 (2019)

15. A.S. Petukhova, I.S. Petukhov, and S.I. Petukhov, ApJ, 880, 17 (2019) 